\title{
Evaluation of adherence to surgical prophylaxis guidelines and expenditure on antimicrobial agents used for surgical prophylaxis
}

\author{
Authors \\ Mahendra M. Gaikwad ${ }^{1}$, Sangeeta S. Dabhade ${ }^{2}$, Balasaheb B. Ghongane ${ }^{3}$ \\ ${ }^{1}$ Junior resident, Dept. of Pharmacology, B. J. Government Medical College and SGH Pune, India-411001 \\ Email: mahendra_gaikwad2006@yahoo.com \\ ${ }^{2}$ Associate Professor, Dept. of Pharmacology, B. J. Govt Medical College and SGH Pune, India-411001 \\ ${ }^{3}$ Professor and Head, Dept. of Pharmacology, B. J. Govt Medical College and SGH Pune, India-411001 \\ Email: ghongane.bb@gmail.com \\ Corresponding Author \\ Sangeeta S. Dabhade \\ Email: sangeetadr99@gmail.com, Contact No.+91-9823840872
}

\begin{abstract}
Background: Surgical site infection (SSI) accounts for $15 \%$ of all nosocomial infections and represents the most common nosocomial infection.

Aims: To evaluate adherence to surgical prophylaxis guidelines and expenditure because of non-adherence to guidelines regarding antimicrobial agents (AMA) used for surgical prophylaxis.

Settings and Design: Prospective observational study in a tertiary care hospital.

Methods and Material: A prospective, observational study was conducted in 600 surgical cases from General surgery, Obstetrics and Gynaecology and Orthopaedics departments in a tertiary care hospital. Data were collected from medical case sheets about AMA used for surgical prophylaxis with regards to dose, timing, frequency, duration and expenditure on AMA. Appropriateness of surgical prophylaxis was evaluated with National treatment guidelines-2016 and hospital antibiotic policy.

Statistical analysis used: Microsoft excel 2013

Results: The Choice of antimicrobial agent was in adherence to guideline in $87.30 \%, 80.30 \%$ and $83.50 \%$ cases and total duration of surgical prophylaxis was in adherence to guidelines in only $15.74 \%, 16.16 \%$ and $0 \%$ cases in General surgery and Obstetrics and Gynaecology and Orthopaedics respectively. This non-adherence leads to additional expenditure on surgical prophylaxis which was Rs. $162.76 \pm 168.50$, Rs. $353.09 \pm 140.00$ and Rs. $130.06 \pm 124.74$ per patient for General surgery, Orthopaedics and Obstetrics and Gynaecology departments respectively.

Conclusions: Prolonged duration of surgical prophylaxis and in some extend inapt choice causes increased costs for the health care system, emphasizing on continued surveillance of surgical prophylaxis practices.

Keywords: Surgical prophylaxis, antimicrobial agents, expenditure, adherence.
\end{abstract}

\section{Introduction}

Surgical site infection (SSI) accounts for $15 \%$ of all nosocomial infections and among surgical patients, represents the most common nosocomial infection. ${ }^{1}$ Approximately 1 million patients have surgical site infections each year in the United 
States, extending the average hospital stay by one week and increasing the cost of hospitalization by 20 percent. This translates to an additional $\$ 1.5$ billion in health care costs annually. ${ }^{2}$

The purpose of surgical prophylaxis is to reduce the incidence of SSI with minimum alteration of normal microbial flora of host. ${ }^{3}$ Proper antibiotic prophylaxis has been shown to be effective in reducing the incidence of surgical site infections and the selection of an appropriate antimicrobial agent (AMA) depends on the pathogen most likely to cause an infection at surgical site. ${ }^{4}$

Approximately $30-50 \%$ of antibiotic use in hospital practice is now for surgical prophylaxis. However, frequently, the antibiotic is either given at the wrong time or continued for too long. ${ }^{5}$

Consequences of SSIs increases the cost of treatment, longer duration of hospital stay and increase use of antimicrobials which can enhance the antimicrobial resistance among the pathogens likely to cause surgical wound infections. ${ }^{6,7}$

Inappropriate usage and prolonged postoperative doses do not provide any added benefit but may increase the incidence of antibiotic resistance. ${ }^{8}$ These type of errors in the surgical prophylaxis for surgical patients are one of the most common types of medication errors in hospitals and there is a necessity to generate baseline data on the pattern of the use of prophylactic antimicrobials. ${ }^{9}$

So the monitoring of prescriptions and drug utilization studies could identify the related problems and provide feedback to prescribers. In a developing countries like India due to availability of limited funds for health care it becomes very important to prescribe drug rationally so that available funds can be optimally utilised. ${ }^{10}$

Hence, this study is planned to evaluate the appropriateness of use of antimicrobial agents for surgical prophylaxis by comparing with standard guidelines for surgical prophylaxis.

Also to get an account of pharmacoeconomics of antimicrobial agents used for surgical prophylaxis so as to find out the expenditure on it and the actual cost burden on the health sector because of inappropriate use of antimicrobial agents for surgical prophylaxis due to non-adherence with the standard guidelines for surgical prophylaxis in relation to antimicrobial agents only.

\section{Subjects and Methods:}

Study design: This was a prospective, observational, hospital based study to evaluate the utilization of antimicrobial agents used for surgical prophylaxis including treatment of postoperative infections. The study was conducted by the Department of Pharmacology, in collaboration with the Departments of General surgery, Orthopaedics and Obstetrics and Gynaecology in a tertiary care hospital.

Study population: Patients undergoing surgeries of clean or clean-contaminated type of surgical wound in the three surgical departments namely General surgery, Orthopaedics and Obstetrics and gynaecology of tertiary care hospital, were screened for the study and subjects who satisfy the inclusion and exclusion criteria mentioned below were recruited for the study.

\section{Inclusion criteria:}

1) Patients undergoing surgeries in surgical department's namely General surgery, Orthopaedics and Obstetrics and Gynaecology.

2) Surgical operations classified as clean (Class I) or clean-contaminated (Class II) according to National Research Center (NRC) Classification

\section{Exclusion criteria}

1) Patients below the age of 18 years. (To exclude minor age group population)

2) Surgical operations classified as contaminated (Class III) or dirty (Class IV) according to NRC Classification.

\section{Detailed research plan}

\section{Data collection}

A prospective, observational study was conducted for a period of six months from July to December 2015 in 600 patients admitted for various surgeries in three surgical departments namely General surgery, Orthopaedics and Obstetrics and gynaecology, after taking official permission from 
above mentioned departments and after approval from Institutional Ethical committee.

The data were collected from medical case sheet (I.P.D. file) and operation notes while the patients were still in the hospital.

The data were collected on a case record form designed for study, includes:

- Demographic details of patients

- Diagnosis, name of surgery done, type of surgery

- Details of Antimicrobial agents (AMA) used for surgical prophylaxis with regards to dose, route, timing of first dose, frequency of administration and total duration of surgical prophylaxis.

Data retrieved from case record forms were entered in Microsoft Excel sheet and assessed for various parameters to find out study objectives.

The appropriateness of surgical antibiotic prophylaxis in the study cases was assessed with regards to choice of AMA, dose of AMA, timing of 1st dose of AMA and total duration of surgical prophylaxis given in study group by comparing the data with following standard guidelines for surgical antibiotic prophylaxis:

1. Local antibiotic policy (LAP) ${ }^{11}$

2. National treatment guidelines (NTG) for antimicrobial used in infectious diseases by National Centre for Disease Control (NCDC), India $^{12}$

3. American Society of Health-System Pharmacists (ASHP): Clinical practical guidelines for antimicrobial prophylaxis in surgery. ${ }^{13}$

Total expenditure on antimicrobial agents used for surgical antibiotic prophylaxis patients were calculated on the basis of cost of each antimicrobial agent given in government rate contract (RC) book from drug store of hospital. Likewise expenditure on individual AMA used and the total expenditure by individual surgical department were calculated. Any increase or decrease in expenditure on AMA used for surgical antibiotic prophylaxis due to non-adherence of surgical antibiotic prophylaxis with the above mentioned standard guidelines with regards to choice, dose, timing of first dose and duration were calculated for the each surgical case and the average increase or decrease in expenditure per surgical case by each of surgical department included in study were calculated to high-lighten the direct cost burden on health care system due to inappropriate antimicrobial administration for surgical prophylaxis by not following the standard guidelines.

\section{Statistical analysis}

For the statistical analysis average, mean and standard deviation (SD) were calculated by using Microsoft Excel 2013.

\section{Results}

Data of total 600 surgical cases, 200 cases from each of the three surgical department's namely General surgery, Orthopaedics and Obstetrics and Gynaecology departments were analysed.

\section{Demographic details}

Table 1: Age, gender and types of surgery included in study from different departments:

\begin{tabular}{|l|c|c|c|c|c|}
\hline \multirow{2}{*}{ Department } & $\begin{array}{c}\text { Age } \pm \\
\text { S.D. }\end{array}$ & \multicolumn{2}{|c|}{ Gender } & \multicolumn{2}{c|}{ Types of surgery } \\
\cline { 2 - 6 } & & Males & Females & Class I & Class II \\
\hline General & $44.85 \pm$ & 138 & 62 & 134 & 66 \\
surgery & 14.37 & $(69 \%)$ & $(31 \%)$ & $(67 \%)$ & $(38 \%)$ \\
\hline Orthopaedics & $44.24 \pm$ & 133 & 66 & 182 & $18(9 \%)$ \\
& 18.00 & $(66 \%)$ & $(33 \%)$ & $(91 \%)$ & \\
\hline $\begin{array}{l}\text { Obstetrics \& } \\
\text { Gynaecology }\end{array}$ & $29.40 \pm$ & $0(0 \%)$ & 100 & 9 & 191 \\
$(18.00$ & & $(100 \%)$ & $(4.5 \%)$ & $(95.5 \%)$ \\
\hline
\end{tabular}

Table 1 shows that mean age of patients was 44.85 $\pm 14.37,44.24 \pm 18.00$ and $29.40 \pm 18.00$ in General surgery, Orthopaedics and Obstetrics \& Gynaecology departments respectively.

With respect to gender there were 138 (69\%) and $133(66 \%)$ males in General surgery and Orthopaedics departments respectively. Also 62 (31\%) and $66(33 \%)$ were females in General surgery and Orthopaedics departments respectively.

Naturally all were females in Obstetrics \& Gynaecology department.

$134(67 \%), 182(91 \%)$ and $9(4.5 \%)$ were class I type of surgeries in in General surgery, Orthopaedics and Obstetrics \& Gynaecology departments respectively.

66 (38\%), 18 (9\%) and 191 (95.5\%) were class II type of surgeries in in General surgery, 
Orthopaedics and Obstetrics \& Gynaecology departments respectively.

Appropriateness of surgical prophylaxis in adherence to standard guidelines for surgical prophylaxis:

A) Choice of antimicrobial agent

Figure 1: Choice of antimicrobial agent (AMA) in adherence to local antibiotic policy (LAP), National treatment guideline (NTG), India and American Society of Health-System Pharmacists (ASHP) guideline.

Choice of AMA in adherence to LAP and NTG India guidelines

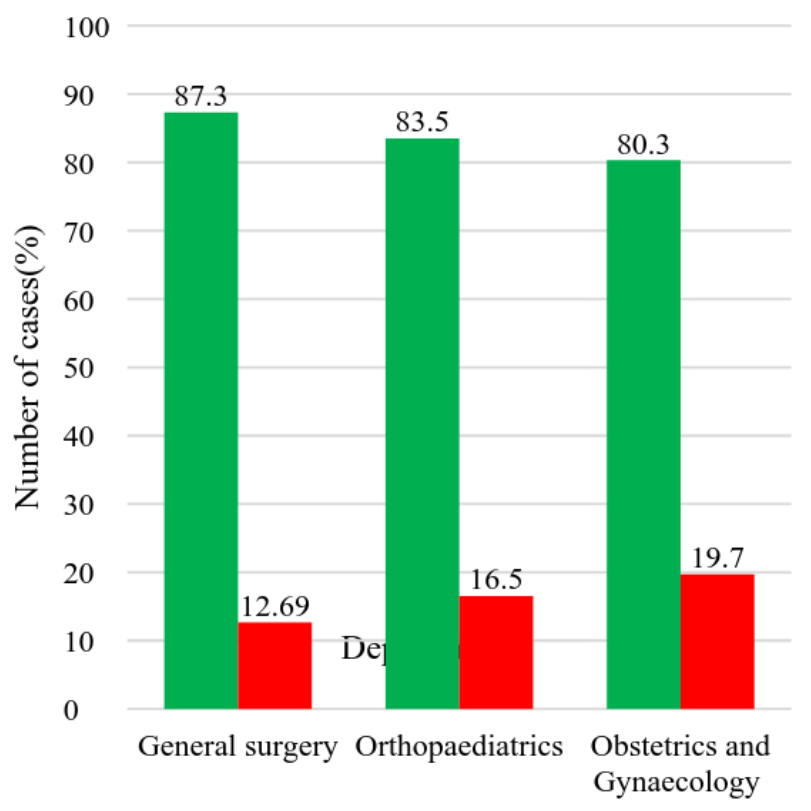

ADHERENCE NON-ADHERENCE

Figure 1 shows the appropriateness of surgical prophylaxis with regards to choice of antimicrobial agents in adherence to local antibiotic policy (LAP), National treatment guideline (NTG), India and American Society of Health-System Pharmacists (ASHP) guideline.

Choice of AMA was in adherence with guidelines in $87.30 \%, 83.50 \%$ and $80.30 \%$ cases of General surgery, Orthopaedics and Obstetrics and Gynaecology departments respectively.

B) Dose of antimicrobial agent:
Figure 2: Appropriateness of $\underline{\text { dose }}$ of antimicrobial agent (AMA) in adherence to local antibiotic policy (LAP), National treatment guideline (NTG), India and American Society of Health-System Pharmacists (ASHP) guideline.

\section{Dose of AMA in adherence to LAP and NTG}

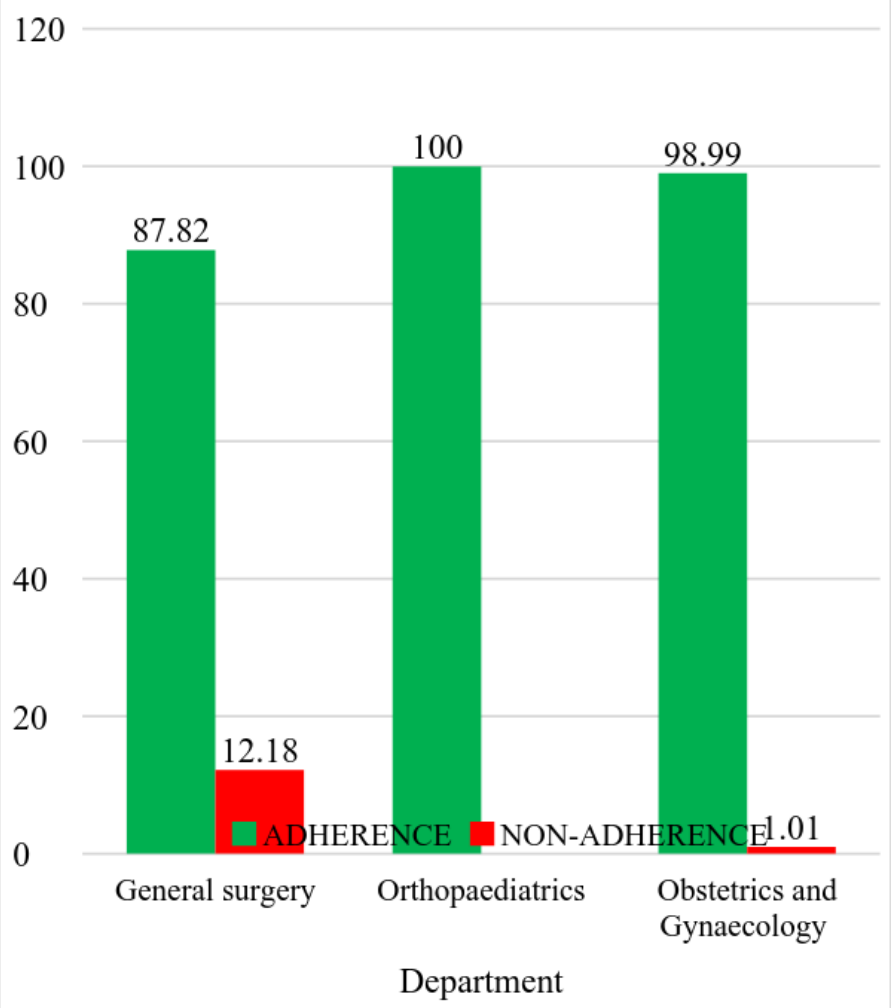

Figure 2 shows the appropriateness of surgical prophylaxis with regards to dose of antimicrobial agents in adherence to local antibiotic policy (LAP), National treatment guideline (NTG), India and American Society of Health-System Pharmacists (ASHP) guideline.

With this regards in General surgery, adherence were seen with $87.81 \%$ cases and non-adherence in $12.18 \%$ cases, in Orthopaedics, adherence to guidelines were seen in all .i.e. $100 \%$ cases and in Obstetrics and Gynaecology, adherence were seen with $98.99 \%$ cases and non-adherence in $1.01 \%$ cases.

C) Timing of first dose of antimicrobial agent used for surgical prophylaxis: 
Figure 3: Timing of first dose of antimicrobial agent in adherence to National treatment guideline (NTG), India and American Society of HealthSystem Pharmacists (ASHP) guideline

Timing of 1 st dose of AMA in adherence to NTG and ASHP

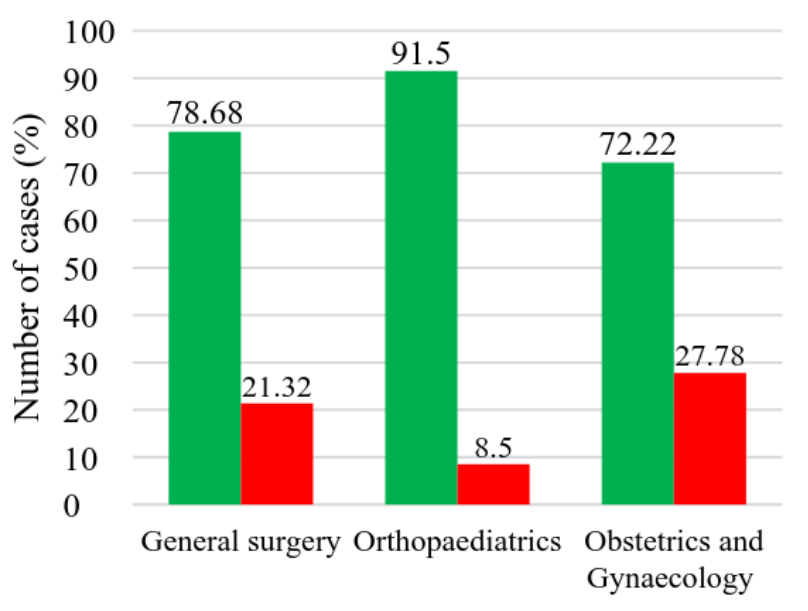

Department

ADHERENCE NON-ADHERENCE

Figure 3 shows appropriateness of timing of first dose of antimicrobial agent in adherence to National treatment guideline (NTG), India and American Society of Health-System Pharmacists (ASHP) guideline.

With this regards in General surgery, adherence were seen in $78.68 \%$ cases and non-adherence in $21.32 \%$ cases.

In Orthopaedics, adherence were seen in $91.50 \%$ cases and non-adherence in $8.50 \%$ cases.

In Obstetrics and Gynaecology, adherence were seen in $72.22 \%$ cases and non-adherence in $27.78 \%$ cases.

D) Total duration of surgical prophylaxis:
Figure 4: Total duration of surgical prophylaxis in adherence to National treatment guideline (NTG), India and American Society of HealthSystem Pharmacists (ASHP) guideline.

Total duration of surgical prophylaxis in adherence to NTG India

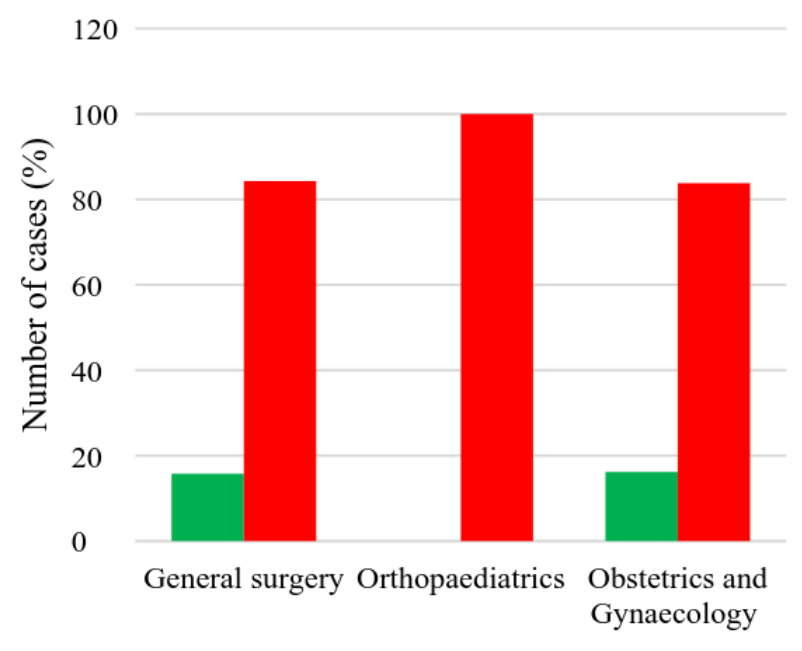

Department

ADHERENCE $\square$ NON-ADHERENCE

Figure 4 shows appropriateness of total duration of surgical prophylaxis in adherence to National treatment guideline (NTG), India and American Society of Health-System Pharmacists (ASHP) guideline.

With this regards in General surgery, adherence were seen in $15.74 \%$ cases and non-adherence in $84.26 \%$ cases, in Orthopaedics, non-adherence to guidelines were seen in all .i.e. in $100 \%$ cases and in Obstetrics and Gynaecology, adherence were seen in $16.16 \%$ cases and non-adherence in $83.83 \%$ cases.

\section{Expenditure on AMA used for surgical prophylaxis:}

Table 2: Total expenditure on AMA used for surgical prophylaxis:

\begin{tabular}{|l|c|}
\hline Department & Total expenditure (Rs.) \\
\hline General surgery & $35,217.15$ \\
\hline Orthopaedics & $75,853.00$ \\
\hline Obstetrics and Gynaecology & $40,285.32$ \\
\hline Total & $1,51,355.50$ \\
\hline
\end{tabular}

Total expenditure on AMA used for surgical prophylaxis by General surgery, Orthopaedics and Obstetrics and Gynaecology were Rs. 35,217.15, Rs. 75,853.00 and Rs. 40,285.32 respectively. 
Table 3: Excess expenditure on surgical prophylaxis due to non-adherence to standard guidelines for surgical prophylaxis

\begin{tabular}{|l|c|c|}
\hline Department & $\begin{array}{c}\text { Average } \\
\text { expenditure on } \\
\text { surgical } \\
\text { prophylaxis per } \\
\text { patient } \\
\text { (Rs) }\end{array}$ & $\begin{array}{c}\text { Average excess } \\
\text { expenditure on } \\
\text { surgical prophylaxis } \\
\text { per patient due to } \\
\text { non-adherence } \\
\text { (Rs) }\end{array}$ \\
\hline General surgery & $218.74 \pm 168.50$ & $162.76 \pm 168.50$ \\
\hline Orthopaedics & $401.34 \pm 140.00$ & $353.09 \pm 140.00$ \\
\hline $\begin{array}{l}\text { Obstetrics and } \\
\text { Gynaecology }\end{array}$ & $232.86 \pm 124.74$ & $130.06 \pm 124.74$ \\
\hline
\end{tabular}

Table 3 shows excess expenditure because of antimicrobial agents used for surgical prophylaxis due to non-adherence to standard guidelines calculated by subtracting average cost of standard surgical prophylaxis from average actual cost on surgical prophylaxis per patient.

For general surgery department average cost of surgical prophylaxis per patient was $218.74 \pm$ 168.50 and excess expenditure per patient due to non-adherence to standard guidelines for surgical prophylaxis was Rs. $162.76 \pm 168.50$.

For orthopaedics department average cost of surgical prophylaxis per patient was $401.34 \pm$ 140.00 and excess expenditure per patient due to non-adherence to standard guidelines for surgical prophylaxis was $353.09 \pm 140.00$.

For Obstetrics and Gynaecology department average cost of surgical prophylaxis per patient was $232.86 \pm 124.74$ and excess expenditure per patient due to non-adherence to standard guidelines for surgical prophylaxis was $130.06 \pm$ 124.74.

\section{Discussion}

This was a prospective, observational and hospital based study conducted in surgical departments namely Generals surgery, Orthopaedics and Obstetrics and Gynaecology of tertiary care hospital with aim to evaluate the utilization and pharmaco-economics of antimicrobial agents used for surgical prophylaxis.

In this study patients above 18 years undergoing clean and clean-contaminated types of surgeries in three surgical departments namely Generals surgery, Obstetrics and Gynaecology and Orthopaedics were included.

In General surgery department maximum number of cases included in study were belonged to age group of $48-57$ i.e. 52 (26\%) cases with mean age of $44.85 \pm 14.33$ (SD) years.

In Orthopaedics department maximum number of cases included belong to age group of 28-37 i.e. $42(21 \%)$ with mean age of $44.24 \pm 17.50(\mathrm{SD})$ years.

In Obstetrics and Gynaecology department maximum number of cases included belong to age group of 18-27 i.e. 120 (60\%) with mean age of $29.40 \pm 10.26$ (SD) years.

A study on surgical prophylaxis pattern in India by Kaur $\mathrm{R}$ et al ${ }^{14}$, mean age was $40.22 \pm$ $15.22(\mathrm{SD})$ and $31.40 \pm 12.98(\mathrm{SD})$ for General surgery and Obstetrics and Gynaecology cases included in study.

In this study male patients were more admitted as compared to female patients in total as well as separately in General surgery and Orthopaedics departments. The reason for more male admissions in this study may be attributed to more male to female ratio in Maharashtra and in the Indian scenario it is noticed that female populations are reluctant to utilize health care facilities even if they are critically ill.

In all 600 cases enrolled in study from General surgery, Orthopaedics and Obstetrics and Gynaecology departments, 325(54.16\%) were clean surgeries and 275(45.83\%) were cleancontaminated surgeries.

In a study by Ramesh A. et al ${ }^{15}, 60 \%$ were clean surgeries and $40 \%$ were clean-contaminated surgeries.

In General surgery and Obstetrics and Gynaecology departments, metronidazole was most commonly used antimicrobial agent for surgical prophylaxis. Metronidazole was used in combination with 3rd generation cephalosporins i.e. either with ceftriaxone in $37.57 \%$ cases from General surgery and in $9.09 \%$ cases from Obstetrics and Gynaecology departments or with cefotaxime in $24.43 \%$ cases from General surgery 
and $71.26 \%$ cases from Obstetrics and Gynaecology departments. But metronidazole was not used as a single drug for surgical prophylaxis in either of above departments.

In Orthopaedics department, amikacin was most commonly used antimicrobial agent for surgical prophylaxis. Amikacin was used in combination with 3rd generation cephalosporins i.e. either with ceftriaxone in $70 \%$ cases or with cefotaxime in $17 \%$ cases but was not used as a single drug for surgical prophylaxis in Orthopaedics surgical procedures.

This study evaluated whether the surgical prophylaxis given in study groups was appropriate and in adherence with local antibiotic policy (LAP), National treatment guideline (NTG), India and American Society of Health-System Pharmacists (ASHP) guideline.

The appropriateness of surgical prophylaxis was evaluated with regards to choice of antimicrobial agent, dose of antimicrobial agent, the timing of administration of first dose of antimicrobial agent in relation to surgical incision and total duration of prophylaxis.

The Choice of antimicrobial agent was in adherence to local antibiotic policy (LAP), National treatment guideline (NTG), India and American Society of Health-System Pharmacists (ASHP) guideline in $87.30 \%, 83.5 \%$ and $80.30 \%$ case of General surgery, Orthopaedics and Obstetrics and Gynaecology departments.

According to ASHP and NTG India, first generation cephalosporin i.e.cefazolin has been recommended as a drug of choice for surgical prophylaxis except for biliary tract and colorectal procedures where ceftriaxone $\left(3^{\text {rd }}\right.$ generation cephalosporin) can be used as alternative. In our study we considered ceftriaxone and cefotaxime as adherence to guidelines as belonging to same class of drugs as mentioned in above guidelines and as cefazoline was not available in study hospital.

A study by Ram VGR et al ${ }^{16}$ on surgical antibiotic prophylaxis in a tertiary care teaching hospital in India, adherence to ASHP guideline with regards to choice of AMA seen in $80 \%$ cases of General surgery which was almost same as finding of this study. In a study by Parulekar et al ${ }^{17}$ appropriateness of choice of AMA in adherence to local antibiotic policy was seen in $68 \%$ of cases.

Local antibiotic policy (LAP), National treatment guideline (NTG), India and American Society of Health-System Pharmacists (ASHP) guideline suggest that standard therapeutic dose of antimicrobial agent by intravenous route is sufficient for prophylaxis.

In this study the dose of antimicrobial agent was in adherence with guidelines in $87.81 \%, 100 \%$ and $98.99 \%$ cases of General surgery, Orthopaedics and Obstetrics and gynaecology department respectively where a standard therapeutic dose of antimicrobial agent by intravenous route was administered for providing surgical prophylaxis.

Non-adherence in $12.18 \%$ and $1.01 \%$ cases of General surgery and Obstetrics and gynaecology respectively was because of administration of dose of antimicrobial agent by oral route which was not justified by guidelines.

In a study by Parulekar et al ${ }^{17}$ appropriateness of dose of AMA in adherence to local antibiotic policy was seen in $75 \%$ of cases

In a study by Prasad $\mathrm{AB}$ et al ${ }^{18}$ dose of antimicrobial agent was in adherence with ASHP guideline in $86 \%$ of cases.

National treatment guideline (NTG), India and American Society of Health-System Pharmacists (ASHP) guideline suggest that for surgical procedures, intravenous prophylactic antibiotics should be given within 60-30 minutes before the skin is incised and as close to time of incision as practically possible.

In this study appropriateness of timing of first dose of antimicrobial agent in adherence to guidelines were seen with $78.68 \%, 91.50 \%$ and $72.22 \%$ cases of General surgery, Orthopaedics and Obstetrics and gynaecology departments respectively. 
In a study by Prasad $A B$ et al ${ }^{18}$ in $75.7 \%$ cases timing of first dose of antimicrobial agent was in adherence to guidelines.

In a study by Parulekar et al ${ }^{17}$ done in tertiary care hospital, Mumbai, appropriateness of timing of first dose of antimicrobial agent in adherence to guidelines were seen with $89 \%$ cases.

National treatment guideline (NTG), India and American Society of Health-System Pharmacists (ASHP) guideline recommended that surgical prophylaxis should be discontinued within 24 hours after surgery. There was no any recommendation in local antibiotic policy about for how much duration prophylactic antimicrobial agents should be continued.

In this study appropriateness of total duration of surgical prophylaxis in adherence to guidelines were seen with $15.74 \%, 0 \%$ and $16.16 \%$ cases of General surgery, Orthopaedics and Obstetrics and gynaecology departments respectively.

In a study done in Turkey by Sozen $\mathrm{H}$ et al ${ }^{19}$ $13.5 \%$ of cases total duration of surgical prophylaxis was in adherence to guidelines.

So in $>80 \%$ of cases surgical prophylaxis was continued beyond 24 hours. The mean duration of surgical antibiotic prophylaxis were $3.24 \pm 1.75$, $4.90 \pm 1.28$ and $4.43 \pm 1.83$ days in General surgery, Orthopaedics and Obstetrics and gynaecology departments respectively.

In a study by Prasad AB et al ${ }^{18}$ none of studied cases shown appropriateness of total duration of surgical prophylaxis in adherence to guidelines.

Askarian $\mathrm{M}$ et al ${ }^{20}$ and Hosoglu S et al ${ }^{21}$ studies also revealed that long duration of surgical prophylaxis is a common practice.

Various studies suggested that short duration prophylaxis is equally effective as longer duration of prophylaxis in preventing surgical site infections. ${ }^{13,22}$

Also most of studies states that extended prophylaxis beyond 24 hours has been shown to be of no benefit. ${ }^{23,24}$

Also according to a study by Ram GRV et al ${ }^{16}$ approximately $30-50 \%$ of antibiotics use in hospital practice is now for surgical prophylaxis, however between $30 \%$ and $90 \%$ of this prophylaxis is inappropriate.

Therefore this study decided to calculate cost expenditure over antimicrobial agents (AMA) used for surgical prophylaxis in study departments and excess expenditure on antimicrobial agents used for surgical prophylaxis because of inappropriateness or non-adherence with standard guidelines for surgical prophylaxis.

Cost of each AMA used for surgical prophylaxis and for treatment of post-operative infections per department were calculated on the basis of prices given in rate contract (RC) book of drug store of the study institution.

Out of total expenditure on AMAs, 63\%, 85\% and $52 \%$ of expenditure was because of AMAs used for surgical prophylaxis in General surgery, Orthopaedics and Obstetrics and Gynaecology departments respectively, indicating that for more than half of total expenditure, AMAs used for surgical prophylaxis were the stakeholders.

Such a high contribution by antimicrobial agents used for surgical prophylaxis was because almost 99\% of cases in study groups received AMAs for surgical prophylaxis and more than one AMA i.e. combination of two or three antimicrobial agents in most of cases for more than 24 hours of duration after surgery was used as discussed above.

When the appropriateness of surgical prophylaxis in adherence with evidence based guidelines for surgical prophylaxis accessed, in majority of cases surgical prophylaxis was more inappropriate with respect to the total duration of prophylaxis as compared with choice, dose of AMA and the timing of first dose which were more consistent with guidelines.

These findings were in consistent with findings of a study done on compliance of surgical prophylaxis with guidelines in tertiary care hospital of Mumbai by Parulekar et at. ${ }^{17}$

Other western world studies also gave similar findings that high frequency of inappropriateness was with respect to the total duration of surgical prophylaxis. ${ }^{25,26}$ 
In this study non-adherence to guidelines mostly with respect to total duration of prophylaxis lead down the continuation of prophylaxis for longer duration which should not be continued beyond 24 hours of surgery as per recommendation.

All of this contributed to excess expenditure on surgical prophylaxis due to non-adherence with guidelines. In this study average excess expenditure on surgical prophylaxis per patient due to non-adherence to guidelines was Rs. $162.76 \pm 168.50$, Rs. $353.09 \pm 140.00$ and Rs. $130.06 \pm 124.74$ for General surgery, Orthopaedics and Obstetrics and Gynaecology departments respectively.

So this excess expenditure on antimicrobial agents used for surgical prophylaxis could be saved if all the surgical prophylaxis were given in strict adherence to standard guidelines with respect to the choice, dose of AMA, timing of first dose and total duration of surgical prophylaxis.

A study by Sozen H et al ${ }^{19}$ also shows that approximate cost on surgical prophylaxis could be reduced up to $90 \%$ when a proper use of antibiotics and proper duration of the applications are adopted in accordance with guidelines.

A study by Mathur $\mathrm{P}$ et al ${ }^{27}$ in trauma center of All India Institute of Medical Sciences (AIIMS), New Delhi, India observed that the average cost of short course treatment in accordance with guidelines amounted to Rs.150 per patient as compared to Rs.1900 per patient for prolonged combinational regimens.

Sasse et al. ${ }^{28}$ also reported that a potential saving of US $\$ 6.1$ million could be made if surgical antimicrobial prophylaxis were given according to recommendations.

Study by Askarian $\mathrm{M}$ et al ${ }^{20}$ found an extra cost of US $\$ 8,332$ because of non-adherence to ASHP guideline with regards to prolonged duration of antimicrobial prophylaxis.

A study by Ozkurt $\mathrm{Z}$ et al ${ }^{29}$ stated that as compared to the developed countries, antimicrobials are overprescribed in developing countries, where an average $35 \%$ of health budget is spent on antibiotics.

\section{References}

1. Watanabe A, Kohnoe S, Shimabukuro R. Risk factors associated with surgical site infections in upper and lower gastrointestinal surgery. Sur Today. 2008; 38:404-12.

2. Woods R K, Dellinger E P.Current Guidelines for antibiotic prophylaxis of surgical wounds. Am Fam Physician 1998; 57:2731-40.

3. Tripathi KD. Essentials of medical pharmacology.7th Ed.New Delhi:Jaypee; 2013. Chapter 49: Antimicrobial drugs: General consideration;Pg:701-05.

4. Rehan HS,Kakkar A,Goel S. Surgical antibiotic prophylaxis in a tertiary care teaching hospital in India.Int $\mathbf{J}$ Infect Contol 2010;06( 2):1-6.

5. Dettenkofer M, Forster DH, Ebner W, gastmeier P, ruden $\mathrm{H}$, Daschner FD. The practice of perioperative antibiotic prophylaxis in eight german hospitals. Infection 2002; 30:164-67.

6. Townsend CM,Beauchamp RD,Evers BM, Mattox KL. Chapter 11: Principles of preoperative and operative surgery. Sabiston Texbook Of Surgery:The biological basis of modern surgical practice; 19th edition;2012; Philadelphia; Elsevier:226-43.

7. Gagliardi A R, Fenech D, Eskicioglu C, Nathens AB, McLeod R. Factors influencing antibiotic prophylaxis for surgical site infection prevention in general surgey: a review of the literature. Can J Surg 2009;52:481-89.

8. Stulberg JJ, Delaney CP, Neuhauser DV, et al. Adherence to surgical care improvement project measures and the association with postoperative infections. JAMA. 2010;303(24):2479-85.

9. Salkind AR,Rao KC. Antibiotics prophylaxis to prevent surgical site infection. Am Fam Physician.2011 Mar 1;85(5):585-90. 
10. Afzal Khan AK, Mirshad PV, Rashed MR. Usage of Antimicrobials for Prevention of Surgical Site Infection (SSI). Journal of Clinical and Diagnostic Research. 2013 ;7(4): 671-74.

11. Antibiotic policy-BJ Medical College. Website:www.bjmcpune.org/Antibiotic\%2 0Policy\%202014.doc

(Citedon 10/01/2016.15:10:30)

12. National Treatment Guidelines for Antimicrobial Use in Infectious Diseases. Version 1.0 (2016).National centre for disease control.

Website:

pbhealth.gov.in/AMR_guideline70014958

89.pdf. (Cited on 10/05/2016.16:20:15)

13. American Society of Health System Pharmacists. The ASHP therapeutic guidelines on antimicrobial prophylaxis in surgery.

Website:http://www.ashp.org/DocLibrary/ BestPractices/TG_Surgical.aspx.(Cited on:13-02-2016.15:32:30.)

14. Kaur R, Salman MT, Gupta NK, Gupta U, Ahmad A, Verma VK. Presurgical antibiotic prophylaxis pattern in an Indian tertiary care teaching hospital. JK Science.2015; 17(2):73-78.

15. Ramesh A, Dharini R. Surgical site infection in teaching hospital.Clinio Microbiological and Epidemiological profile. Int J Biol Med Res.2012; 3(3):2050-2053.

16. Ram VGR, VidyaSagar R.A, Krishnan GG, Senthivelan M. Surgical antibiotic prophylaxis in a tertiary care teaching hospital in India. World journal of pharmacy and pharmaceutical sciences 2014;3: 968-76.

17. Parulekar L, Soman R, Singhal T, Rodrigues C, Dastur FD, Mehta A. How good is compliance with surgical antibiotic prophylaxis guidelines in a tertiary care private hospital in India? A prospective study. Indian J Surg 2009;71:15-18.
18. Prasad AB, Pore SM. Adherence to guidelines for antibiotic prophylaxis in major elective surgeries in a tertiary care government and a co-operative hospital. Pharmacie Globale (IJCP) 2013;08 (04):15.

19. Sozen H, Cetinkaya M, Murat S, Caylak SD, hazer DB, Canbek U et al. Cost and efficacy of perioperative antibiotic prophylaxis in a state hospital in turkey. Acta Medica Mediterr. 2013;29:455-60.

20. Askarian M, Moravveji AR, Mirkhani H, Namazi S, Weed H. Adherence to American Society of Health-System Pharmacists surgical antibiotic prophylaxis guidelines in Iran. Infect Control Hosp Epidemiol. 2006; 27: 876-878.

21. Hosoglu S, Sunbul M, Erol S, Altindis M, Caylan R, Demirdag K et al. A national survey of surgical antibiotic prophylaxis in Turkey. Infect Control Hosp Epidemiol.2003; 24: 758-761.

22. Mangram AJ, Horan TC, Pearson ML. Guideline for prevention of surgical site infection, 1999. Hospital Infection Control Practices Advisory Committee. Infect Control Hosp Epidemiol 1999; 20:250-78.

23. Bailly P, Lallemand S, Thouverez M, Talon D. Multicentre study on the appropriateness of surgical antibiotic prophylaxis. J Hosp Infect.2001; 49: 135138.

24. Vaisbrud V, Raveh D, Schlesinger Y, Yinnon AM.Surveillance of antimicrobial prophylaxis for surgical procedures. Infect Control Hosp Epidemiol .1999;20: 610613.

25. Al-Momany NH, Al-Bakri AG, Makahleh ZM, Wazaify MMB. Adherence to international antimicrobial prophylaxis guidelines in cardiac surgery:a Jordanian study demonstrates need for quality improvement. J Manag Care Pharm.2009;15(3):262-71. 
26. Lallemand S, Thouverez M, Bailly $P$, Bertrand X, Talon D. Non-observance of guidelines for surgical antimicrobial prophylaxis and surgical-site infections. Pharm World Sci.2002;24(3):95-9.

27. Antimicrobial prophylaxis for surgery. Treat Guidel Med Lett. 2009;7(82):47-52.

28. Sasse A, Mertens R, Sion JP, Ronveaux O, Bossens M, Mol PD, et al. Surgical prophylaxis in Belgian hospitals: estimate of costs and potential savings. J Antimicrob Chemother.1998;41(2):26772.

29. Ozkurt Z, Erol S, Kadanali A, Ertek M, Ozden K, Tasyaran MA. Changes in antibiotic use, cost and consumption after an antibiotic restriction policy applied by infectious disease specialists. Jpn J Infect Dis 2005 ; $58: 338-43$. 